grunde liegenden Absorptionskurven von Desoxycholsäure- und Chenodesoxycholsäuregemischen gut reproduzierbar, wie auch Wiederauffindungsversuche entsprechender Mengen der beiden Dihydroxycholansäuren die Brauchbarkeit des Verfahrens bestätigen.
Uber die Anwendung dieser Methode an biologischem Material und die Analyse der dünnschichtchromatographisch ebenfalls nicht zu trennenden Dihydroxycholansäurekonjugate des Taurins und des Glycins wird gesondert berichtet.

\title{
Literatur
}

1. Gänshirt, H., F. W. Koss und K. Morianz, ArzneimittelForsch., Aulendorf 10, 943 (1960). - 2. HormanN, A. F., J. Lipid Res. 3, 127 (1962). - 3. FrosCH, B. und H. WAGENER, diese Z., im Druck. - 4. Hamilton, J. G., Gas-Chrom Newsletter 4, 1
(1963). - 5. ENERoTh, P., J. Lipid Res. 4, 11 (1963). - 6. NoRman, A., Acta chem. scand. 7, 1413 (1953). - 7. Carey, B. B., J. Clin. Invest. 37, 1944 (1958). - 8. Wootron, I. D. P. und H. S. WIGGINs, Biochem. J. 55, 292 (1953).
Dr. med. Bruno Frosch

II. Medizinische Klinik und Poliklinik der Freien Universität Berlin

1 Berlin 19, Spandauer Damm 130

\section{Über die Bestimmung von glykolytischen Fermenten beim Krebskranken}

\author{
Von \\ W. Lührs und K. Chrometzka \\ Aus dem Sanatorium „Bergfrieden" - Rottach-Egern der Arbeitsgemeinscbaft für Krebsbekämpfung Sitz Bocbum - \\ Rubrknappscbaft (Chefarzt: Prof. Dr. W. Lübrs)
}

Herrn Professor Warburg zum 80. Geburtstag in dankbarer Verehrung gewidmet

(Der Schriftleitung zugegangen am 22. Juni 1963)

\begin{abstract}
Die heute in der Klinik gebräuchlichen Fermentbestimmungen werden aufgezeigt. Es werderi die Serumfermentwerte bei 144 Krebskranken ausgewertet. Der Effekt einer Behandlung bei Krebskranken kann durch die Fermentwerte objektiviert werden. Als neuestes Ferment wurde die Glutathionreduktase-Aktivität im Serum bestimmt. Bei 303 Patienten, die wegen eines Malignoms operiert und/oder bestrahlt worden waren, wurden die Aldolase, Milchsäuredehydrogenase und die Glutathionreduktase bestimmt. Hierbei zeigte sich beim Vorhandensein von Rezidiven oder Metastasen ein schnelleres Ansprechen der SGR auf diesen pathologischen Befund.
\end{abstract}

\begin{abstract}
The customary enzyme dèterminations currently used in clinical investigation are given. Serum enzyme levels are evaluated for 144 cancer patients. The effect of treatment on cancer patients can be made objective by these enzyme values. Themost recent enzyme test measures serum glutathione reductase. Aldolase, lactic acid dehydrogenase and glutathione reductase were measured in 303 patients who had been operated on and/or irradiated for cancer. SGR showed the most rapid response to relapses or metastases.
\end{abstract}

In zunehmendem Maße werden heute Fermentaktivitäten im Serum zur klinischen Diagnostik benutzit. Neben der Bestimmung der Fermente in Exkreten oder Körperflüssigkeiten hat der Fermentnachweis im Blut an Bedeutung gewonnen. Er kann unter gewissen Umständen ein gutes $\mathrm{Ma} ß$ für das Vorliegen und die Ausbreitung, damit die Progredienz $(1,2)$ bestimmter Tumoren abgeben. Es muß aber betont werden, daß es sich nicht um sogenannte "spezifische Krebsteste" handelt, sondern um Nachweismethoden, die uns bei der Differentialdiagnose helfen, über latente oder progrediente Phasen der Krebskrankheit etwas auszusagen und eine objektive Auswertung der Therapie $(1,2)$ ermöglichen. - Der älteste Fermentnachweis, die Diastasebestimmung im Blut und im Urin ist über 50 Jahre alt. Ebenso ist die Bestimmung der Phosphatasen seit 30 Jahren im klinischen Gebrauch. Für die Klinik ist, auch im Hinblick auf ein karzinomatöses Geschehen die Bestimmung der alkalischen und sauren Phosphatase noch heute wichtig.

Die alkalische Serumphosphatase ist ein Ferment, das aus organischen Verbindungen Phosphorsäure abspaltet. 
Das pH-Optimum liegt zwischen 8,9-9,6. Der größte Gehalt an alkalischer Phosphatase läßt sich in den Dünndarmepithelien, in den Nieren, im Lymphgewebe und in den weißen Blutzellen nachweisen. Besonders hoch ist die Konzentration dieses Fermentes im Serum bei wachsenden Knochen und bei benignen und malignen osteoblastischen Knochentumoren. - Bei der Beobachtung von Leberkrankheiten zeigen Erhöhungen der alkalischen Phosphatase jeweils Verschlimmerungen an. Dagegen finden sich bei der Hepatitis, besonders in den ersten 14 Tagen kaum erhöhte Fermentwerte. Vor einigen Jahren konnten wir an einem größeren Krankengut nachweisen (3), daß in einem sehr großen Prozentsatz Krebsmetastasen in der Leber durch eine erhöhte alkalische Serumphosphatase angezeigt werden. In der Kombination mit anderen Fermentbestimmungen hat sich die Bestimmung der alkalischen Phosphatase auch heute noch als wertvoll erwiesen.

Die zweite Gruppe der Phosphatasen bezeichnet man allgemein als saure Pbosphatase, wobei das Optimum der

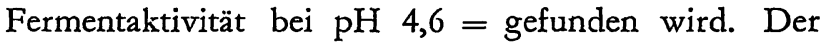
Nachweis dieses Fermentes ist gegenwärtig ein zuverlässiger Test zur „relativen“ Früherfassung eines metastasierenden Prostatakarzinoms. Da beim noch nicht metastasierenden Prostatakarzinom äußerst selten ein erhöhter saurer Phosphatasegehalt im Serum gefunden wird, kann man annehmen, daß erst bei Durchbrechung der Prostatakapsel durch den Tumor und der Absiedlung von Metastasen saure Phosphatase in das Serum übertreten kann. Die Verfolgung der sauren Serumphosphatase ist ein wichtiger Maßstab für die Behandlung mit gegengeschlechtlichen Hormonen.

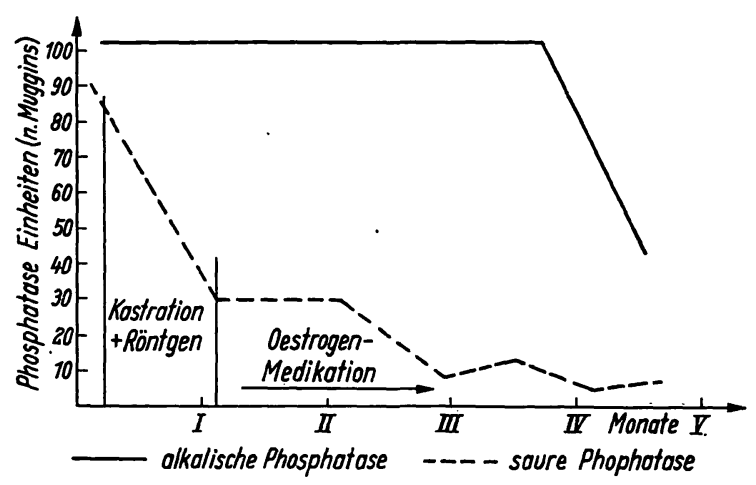

Abb. 1

An einigen Kliniken, besonders im anglo-amerikanischen Raum wird bei Kranken mit Prostatakarzinom und eventuellen Knochenmetastasen obendrein die Phosphogluko-Isomerase oder Phosphohexo-Isomerase bestimmt. Eine Erhöhung dieses Fermentes findet sich ebenfalls bei Knochenmetastasen unid mitunter bei Störungen der Leberzellfunktionen. Da aber der Ausfall dieser Reaktion sehr wechselnd ist, wird dieser Enzymbestimmung nicht allzu große Bedeutung zuerkannt.

Durch Arbeiten von WARBuRg (4) während der letzten 30 Jahre wurden die Reaktionsstufen der Glykolyse und die hier wirksamen Fermente eingehend studiert und aufgeklärt. Methodische Angaben aus experimentellen Studien konnten vom klinischen Laboratorium übernommen werden $(4,5)$. Die s. Z. im Tierversuch gewonnenen Erkenntnisse konnten erst Jahre später in der Klinik bestätigt und von ihr übernommen werden. Heute glaubt man, daß für die Zunahme der Serumkonzentration bekannter glykolytischer Fermente abnorme Syñthese und gleichzeitiger Zellzerfall Voraussetzung ist. Bekannt ist, daß der Stoffwechsel eines Malignoms mit sehr starker Glykolyse einhergeht. Somit war es naheliegend, die Bestimmung glykolytischer Fermente auch zur Karzinomdiagnostik heranzuziehen.

Von den Fermenten, die ziemlich frühzeitig zur Krebsdiagnose herangezogen worden sind, hat die Milchsäuredebydrogenáse (Lactic-acid-dehydrogenase $=\mathrm{LDH}$ ) eine gewisse Bedeutung erlangt. HILL und LEVY (6) zeigten zuerst, daß die Plasmakonzentration dieses Fermentes bei Patienten mit fortgeschrittener Karzinomatose verhältnismäßig häufig erhöht ist. Dieses Ferment wurde im Jahre 1919 von MeYerhof (7) entdeckt. Die Milchsäuredehydrogenase katalysiert die Reaktion Brenztraubensäure $\leftrightharpoons$ Milchsäure. Neben der bereits erwähnten Erhöhung bei Karzinomkranken, findet sich ebenfalls ein erhöhter Serumwert bei Leberzellnekrosen, in der diabetischen Azidose, bei zerebralen Infarktionen und mitunter bei Herzinfarkten. Wir haben nach der ersten Mitteilung von Hill und LEVY an einer größeren Zahl von Kranken mit einem Bronchialkarzinom den Ausfall der Fermentwerte nachgeprüft. Wir konnten damals feststellen, daß man nicht von einer krebsspezifischen Aussage dieser Fermentbestimmung sprechen kann (8). Interessant ist aber auch, daß in karzinomatösen pleuralen und peritonalen Exsudaten häufig ein höherer Wert für die Milchsäuredehydrogenase gefunden werden kann. Bei nicht karzinombedingten Exsudaten lagen die Werte meistens niedriger.

Als weiteres glykolytisches Ferment ist die Aldolase in ihrem Aussagewert für den Kliniker wichtig geworden. Die FDP-Aldolase ist ein in den intermediären Kohlehydratstoffwechsel eingreifendes Ferment, das Fruktose-Diphosphorsäure in zwei Moleküle Triosephosphat spaltet. Warburg und Christian (5) fanden bereits 1943, daß dieses Ferment aus Tumoren in das Blut übertreten kann. Diese ersten Beobachtungen wurden später von Sibley und LEHNiNGER (9) bei Menschen bestätigt. Eine Analyse der Plasmaenzymkonzentration ergab, daß aber nur bei etwa $20 \%$ der Krebsträger eine deutliche Zunahme nachzuweisen ist. Weiterhin kann man eine deutliche Erhöhung der Aldolase bei der akuten Hepatitis und bei der progressiven Muskeldystrophie finden. Bei Leberzirrhose, also bei chronischem Leberschaden und beim Verschlußikterus kann die Aldolase leicht erhöht sein, mitunter findet man aber auch normale Werte. Beim Prostatakarzinom findet sich im Gegensatz zur benignen Prostatahypertrophie eine Erhöhung der Serumaldolase. 
In einer Utbersicht, die wir 1960 veröffentlicht haben (1), kamen wir zu Beobachtungen, die in Abbildung 2 erläutert werden.
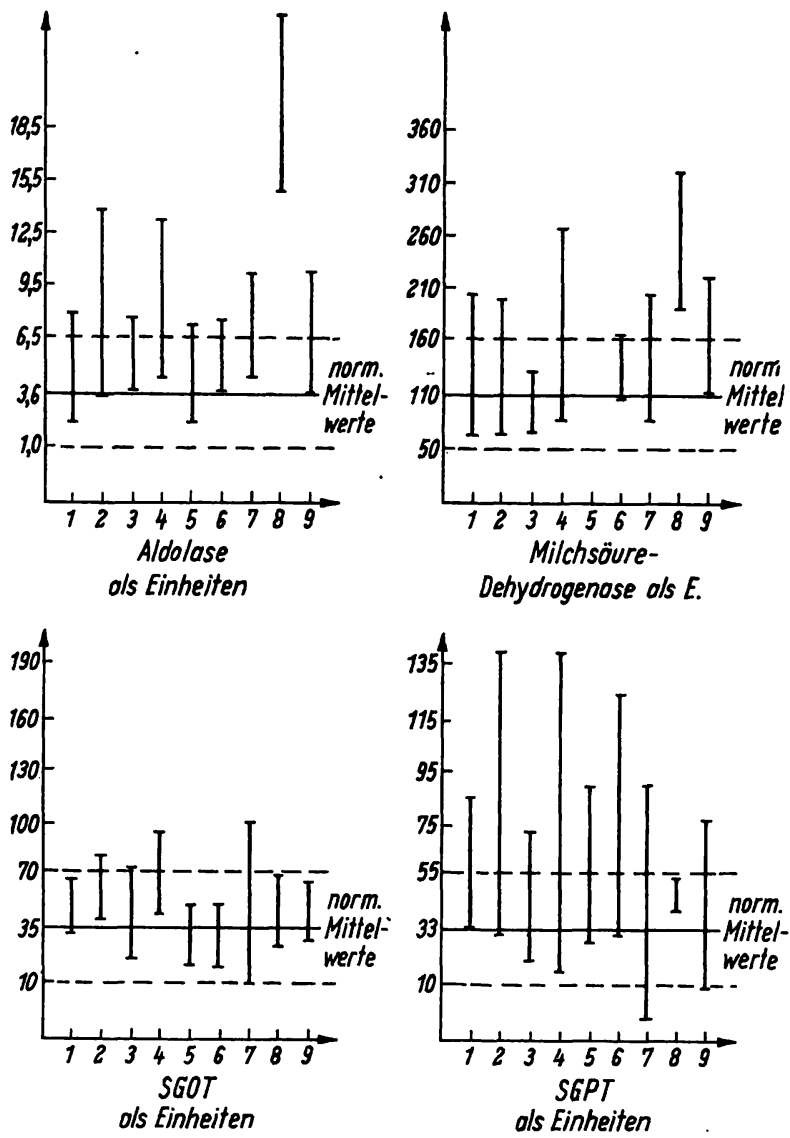

Abb. 2

Durchschnittliche Fermentwerte mit Streuungsbreite bei Kranken mit

1. 24 Bronchial-Ca (isol. Rundherd)

2. 26 Bronchial-Ca mit sekund. Atelektasen usw.

3. 17 Mamma-Ca I-II

4. 19 Mamma-Ca III-IV

5. 10 Magen-Ca postgastrektomiam

6. 13 Magen-Ca palliativ operiert

7. 20 Lymphogranulomatosen

8. 10 chron. myel. Leukämie

9. 15 chron. lymphat. Leukämie

Eine signifikante Erhöhung fand sich bei der myeloischen Leukämie sowohl für die Aldolase als auch für die Milchsäuredehydrogenase. Fortgeschrittene Karzinome ließen für die ebengenannten Enzymnachweise in größerem Ausmạß eine Erhöhung erkennen.

Erwähnt wurde bereits die Phosphogluko-Isomerase, die den reversiblen Reaktionsschritt des Glukose-6-phosphates zu Fruktose-6-phosphat katalysiert. Bereits 1953 fand BoDANSKY (10) nicht nur beim Prostatakarzinom sondern auch beim metastasierenden Mammakarzinom, und bei anderen Tumoren häufig einen erhöhten Serumwert dieses Fermentes. In vielen Fällen bestand eine auffallende Parallele im Anstieg der Phosphogluko-Isomerase und der alkalischen Phosphatase. $\mathrm{Zu}$ ähnlichen Beobachtungen kamen BRUNS und JAKOB (11) sowie Bruns und HINSBERg (12). Bei Krebskranken, die therapeutisch gut beeinflußt werden konnten, stellten Rosotro und RuFro (13) gelegentlich ein gleichzeitiges Abfallen der erhöhten PhosphoglukoIsomerase- und der Aldolasewerte fest.

Soll oder muß eine hormonelle Nachbehandlung durchgeführt werden, so gilt die Faustregel, bei Patientinnen mit einem Mammakarzinom erst etwa zehn Jahre nach der Klimax, etwa nach dem 60. Lebensjahr, Oestrogene $\mathrm{zu}$ verabreichen. Jüngere Patientinnen sollen mit Androgenen behandelt werden. Nun treten aber um das 60. Lebensjahr besonders gehäuft MammakarzinomRezidive und -Metastasen auf. Hier ergibt sich häufig die Schwierigkeit, die wirksame Hormonart, Androgene oder Oestrogene, herauszufinden. Denn äußere Asspekte in Richtung eines beginnenden Virilismus, oder auf der anderen Seite einer noch bestehenden stärkeren Oestrogenstimulierung sind sehr ungenau. Abstriche aus der Vagina, um aus dem Schollenstadium abgeschilferter Epithelien etwas über eine noch vorhandene oestrogene Wirksamkeit auszusagen, Bestimmung der im Urin ausgeschiedenen Hormonmengen und die Resultate von Hormonuntersuchungen im Serum lassen keine exakten Aussagen $\mathrm{zu}$, welches Hormon eingesetzt werden muß. - Hier ergab sich für uns auf Grund eines reichhaltigen klinischen Materials die Erkenntnis, aus dem Ausfall der Aktivitäten die Aldolase und der Milchsäuredehydrogenase schon nach drei bis vier Tagen einer hormonellen Belastung entweder mit Androgenen oder mit Oestrogenen mit Sicherheit aussagen zu können, ob das eine oder das andere Hormon gerechtfertigt war. Es fand sich nämlich eine signifikante Erhöhung bzw. Erniedrigung der Enzyme, sofern das Hormon kontraindiziert oder indiziert war. Die nachstehende Tabelle 1 zeigt, auf Grund der erhaltenen Fermentwerte nach wenigen Tagen, ob das richtige Hormon gewählt worden war. Im ersten Fall (M. H.) findet sich nach einer sechstägigen Belastung mit täglich $50 \mathrm{mg}$ Testoviron ein Absinken der Fermentwerte besonders für die Aldolase und die Milchsäuredehydrogenase. Im Fall J. S. ist deutlich erkennbar, daß die. Verabreichung von Progynon hier kontraindiziert war.

Tab. 1

Fermentaktivitäten vor und nach Hormonbehandlung

\begin{tabular}{|c|c|c|c|c|c|c|}
\hline \multirow{3}{*}{$\begin{array}{c}\text { Ferment } \\
\begin{array}{c}\text { Aldolase } \\
(3,6)\end{array}\end{array}$} & \multirow{2}{*}{\multicolumn{2}{|c|}{$\begin{array}{l}\text { M. H. } \\
\text { Mamma-Ca IV } \\
\text { vor nach } \\
6 \text { Tagen Be- } \\
\text { lastung mit täg- } \\
\text { lich } 50 \mathrm{mg} \\
\text { Testoviron }\end{array}$}} & \multirow{2}{*}{\multicolumn{2}{|c|}{$\begin{array}{c}\text { J.S. } \\
\text { Mamma-Ca IV } \\
\text { vor nach } \\
3 \text { Tagen Bc- } \\
\text { lastung mit täg- } \\
\text { lich } 5 \mathrm{mg} \\
\text { Progynon }\end{array}$}} & \multirow{2}{*}{\multicolumn{2}{|c|}{$\begin{array}{c}\text { Oe. D. } \\
\text { Mamma-Ca IV } \\
\text { vor nach } \\
4 \text { Tagen Be- } \\
\text { lastung mit täg } \\
\text { lich } 100 \mathrm{mg} \\
\text { Testoviron }\end{array}$}} \\
\hline & & & & & & \\
\hline & 12,0 & 3,6 & 8,5 & 17,5 & 11,5 & 4,8 \\
\hline SGOT (35) & 73,0 & 54,0 & 91,0 & 63,0 & 135,0 & 46,0 \\
\hline SGPT (33) & 27,0 & 35,0 & 100,0 & 100,0 & 100,0 & 96,0 \\
\hline $\begin{array}{c}\text { MDH E } \\
\text { (110) }\end{array}$ & 99 & 57 & 208 & 353 & 122 & 54 \\
\hline
\end{tabular}


1957 hat BoDANSKY (14) zusammenfassend über die Veränderungen der Phosphogluko-Isomerase und ihre Relationen zur Phosphoglukomutase bei metastasierenden Tumoren berichtet. Dieses letztere Ferment katalysiert den Reaktionsschritt zwischen Glukose-1phosphat und Glukose-6-phosphat.

Durch die Differenzierung eines ubiquitären Zellenzyms, nämlich der $\mathrm{LDH}$ in seine mehr oder weniger spezifische "Isoenzyme“ versucht man der Organdiagnose näher zu kommen. Es hat sich nämlich herausgestellt, da $\beta$ es verschiedene Formen dieses Enzyms geben kann, die alle dieselbe Reaktion katalysieren, also substrat-spezifisch sind, sich aber hinsichtlich verschiedener Eigenschaften, wie elektrophoretischer Wanderungsgeschwindigkeit, Haftung an Zellulose, Wirkungsoptimum, Hemmbarkeit durch Toxine oder Temperaturveränderung unterscheiden. Auf diese Weise läßt sich eine Steigerung der Organspezifität auch ubiquitär vorkommender Enzyme für die Organdiagnostik erreichen. Für die LDH sind zum Beispiel bis zu fünf solcher Isoenzyme nachgewiesen worden (15). Ebenso fand BraUN (16) eine Heterogenität der Enolase, eines Fermentes, das die Reaktionsstufen zwischen 2-Phosphor-glyzerinsäure und Phosphor-enolbrenztraubensäure reversibel katalysiert. Der Autor fand bei der Enolase eine gleiche Isoenzym-Verteilung in Leber und Niere, die sich aber von der des Herzmuskels, als auch von der des Skelettmuskels deutlich unterschied. Vor einiger Zeit teilten MaNsow und Woblewskr (17) mit, daß sie die Glutathionreduktase-Aktivität im Serum bei krebskranken Menschen sehr häufig erhöht gefunden hatten. Die Glutathionreduktase reduziert oxydiertes Glutathion (GSSG), wobei sie reduziertes
Triphosphorpyridinnukleotid (TPN.H) als Wasserstoffdonator benötigt:

$$
\text { TPN.H }+ \text { GSSG }+\mathrm{H} \leftrightharpoons \mathrm{TPN}+2 \mathrm{GSH} \text {. }
$$

Die Bestimmung wurde ähnlich den bisher bekannten Enzymbestimmungen nach WARBRUG (4) spektrofotometrisch durchgeführt. Interessant war, daß im Gegensatz zu Lebererkrankungen die SGR bei Karzinomkranken sehr häufig erhöht gefunden wurde. Andere Autoren fanden auch nicht regelmäßig eine Erhöhung der SGR-Aktivität bei karzinomkranken Patienten (18). - Für unser klinisches Sanatorium ergibt sich ständig die Aufgabe, Lokalrezidive und erkennbare oder okkulte Metastasen festzustellen und zu objektivieren. Zur Objektivierung des Befundes bei Patienten, die nach einer Operation und/oder Bestrahlung zur Nachbehandlung in unserem klinischen Sanatorium für sechs Wochen beobachtet werden konnten, waren Fermentuntersuchungen in vielen Fällen aufschlußreich. Die folgende Tabelle zeigt 303 lege artis behandelte Karzinomkranke, die bei Abschluß eines sechswöchigen Kuraufenthaltes folgende Enzymmuster besaßen(Tab.2):

In der Auswertung kamen wir zu folgenden Aussagen:

a) Bei 33 Patientinnen, die nach einem operierten und nachbestrahlten Mammakarzinom klinisch frei von Rezidiven und Metastasen waren fanden sich immerhin zwei Frauen mit erhöhter Aldolase und $11 \mathrm{mit}$ ethöhter SGR. Inwieweit diese 11 Patientinnen in der nächsten Zeit ein erneutes Krebswachstum erkennen lassen, wird die Nachsorge erweisen. Interessant sind die Zahlen der Patientinnen, bei denen sich einmal lentikuläre Hautmetastasen, zum anderen aber auch bereits ossale Absiedlungen nachweisen ließen. Hierbei fand sich bei 10 von 15 Patientinnen der SGR-Test erhöht. Die bereits nachweisbaren Metastasen hatten den Organismus innerhalb einer gröberen Umgebungsreaktion so

Tab. 2

Fermentwerte bei 363 behandelten Krebskranken

\begin{tabular}{|c|c|c|c|c|c|c|c|c|c|c|c|c|c|c|c|c|}
\hline Fermente & \multicolumn{2}{|c|}{$\begin{array}{l}\text { Mamma-Ca } \\
\text { frei }\end{array}$} & $\begin{array}{l}\text { Mage } \\
\text { Darn } \\
\text { frei } \\
\text { von } \\
\text { Rez. + }\end{array}$ & $\begin{array}{l}\text { n-Ca } \\
\text { n-Ca } \\
\text { mit } \\
\text { - Meta. }\end{array}$ & \multicolumn{2}{|c|}{$\begin{array}{l}\text { Lymphogra- } \\
\text { nulomatose } \\
\text { frei }\end{array}$} & \multicolumn{2}{|c|}{$\begin{array}{l}\text { Uterus-Ca } \\
\text { Ovarial-Ca }\end{array}$} & $\begin{array}{l}\text { Bronc } \\
\text { frei } \\
\text { von } \\
\text { Rez. }\end{array}$ & $\begin{array}{l}\text { mit } \\
\text { - Meta. }\end{array}$ & latent & $\begin{array}{l}\text { pro- } \\
\text { gred. }\end{array}$ & \multicolumn{2}{|c|}{$\begin{array}{l}\text { übr. Epithel.- } \\
C_{a} \\
\text { frei }\end{array}$} & \multicolumn{2}{|c|}{$\begin{array}{l}\text { übr. Mesen- } \\
\text { chym.-Ca } \\
\text { frei }\end{array}$} \\
\hline $\begin{array}{l}\text { norm. } \\
\text { Aldolase (3-8) }\end{array}$ & 32 & 15 & 57 & 9 & 63 & 17 & 6 & 0 & 19 & 5 & 5 & 5 & 35 & 14 & 13 & 8 \\
\hline erhöht & 2 & 2 & 3 & 5 & 2 & 5 & 0 & 1 & 0 & 4 & 1 & 2 & 1 & 3 & 0 & 2 \\
\hline $\begin{array}{l}\text { norm. } \\
\text { LDH }(200-500)\end{array}$ & 33 & 15 & 60 & 9 & 60 & 19 & 6 & 1 & 13 & 7 & 5 & 2 & 32 & 11 & 17 & 8 \\
\hline exhöht & 0 & 0 & 0 & 3 & 0 & 6 & 0 & 0 & 1 & 2 & 1 & 3 & 1 & 4 & 0 & 2 \\
\hline $\begin{array}{l}\text { norm. } \\
\text { SGR }(40 \pm 15)\end{array}$ & 22 & 5 & 41 & 3 & 42 & 8 & 5 & 0 & 13 & 0 & 4 & 1 & 34 & 5 & 12 & 9 \\
\hline erhöht & 11 & 10 & 16 & 9 & 13 & 18 & $i$ & 1 & 6 & 9 & 1 & 2 & 8 & 12 & 5 & 2 \\
\hline $\begin{array}{l}\text { norm. } \\
\text { GR i. PI. }(36+10)\end{array}$ & 6 & 2 & 10 & 1. & 8 & 4 & 2 & 0 & 2 & 0 & 1 & 1 & 7 & 3 & 3 & 2 \\
\hline erhöht & 0 & 2 & 2 & 1 & 1 & 0 & 0 & 0 & 0 & 0 & 0 & 0 & 2 & 1 & 0 & 0 \\
\hline $\begin{array}{l}\text { norm. } \\
\text { alk. P.ase }(0,9-4,1)\end{array}$ & 15 & 3 & 32 & 1 & 11 & 1 & 4 & 0 & 4 & 2 & 0 & 1 & 6 & 3 & 3 & 3 \\
\hline erhöht & 1 & 1 & $\begin{array}{c}3 \\
\text { schwange }\end{array}$ & 2 & 0 & 4 & 0 & 0 & 1 & 0 & 0 & 0 & 0 & 0 & 0 & 3 \\
\hline
\end{tabular}


wenig beeinflußt, daß Aldolase und LDH kaum beeinflußt wurden, ganz im Gegensatz zu primären unbehandelten Mammakrebsen.

b) Beim Magen-Darm-Karzinom liegen die Verhältnisse ähnlich. Auch hier läßt sich in einem weit erhöhten Prozentsatz eine vermehrte SGR bei den Patienten nachweisen, bei denen Lokalrezidive und. Metastasen klinisch festgestellt werden konnten. Wesentlich erscheint die Zahl von 16 Patienten, die ohne klinischen Befund eine erhöhte SGR aufwiesen. Auch hier wird die nachgebende Fürsorge letztendlich auszusagen vermögen.

c) Bei Kranken mit einer Lymphogranulomatose exlebt man häufiger als bei anderen Tumorarten Rezidive und lymphangitische Metastasen. Somit verwundert es nicht, daß bereits klinisch-phaenotypisch gesunde Lymphogranulomatosen in 16 von 60 Fällen eine ethöhte SGR aufwiesen und eine nachweisbare Progredienz der Lymphogranulomatose in neun Fällen bei 13 Neuerkrankten ebenfalls exhöhte SGR-Werte aufzeigten. Die Aldolase- und LDH-Werte fanden sich in einem weit geringeren Prozentsatz erhöht. Gerade die hohe Zahl von 16 Patienten(innen) mit dem erhöhten SGR-Wert, die z. B. klinisch frei von Rezidiven und Metastasen befunden wurden, werden mit großer Wahrscheinlichkeit eine Progredienz ihres Tumorgeschehens aufweisen. Die klinische Erfahrung macht dies ebenfalls wahrscheinlich.

d) Die Uberlebenszeiten eines Korpus-Kollum-Portio-Karzinoms sind in neuester Zeit so angestiegen, daß Nachkurpatientinnen meist als geheilt betrachtet werden können. Dies bestätigt auch unser Krankengut. Patientinnen nach einem behandelten Ovarialkarzinom finden sich dann ein, wenn eine radikale Behandlung in nicht sehr vielen Fällen zumindest eine "temporäre Heilung" aufweist. e) Zur Nachkurbehandlung kommen etwa $4 / 5$ von operativ behandelten und $1 / 5$ durch ${ }^{60} \mathrm{Co}$-bestrahlten Patienten wegen eines Broncbialkarzinoms. Interessant ist hier bei den neun Kranken mit einem Lokalrezidiv und kontralateralen Metastasen, daß wegen einer stärkeren umgebungsgebundenen Beeinflussung in fast der Hälfte der Fälle die Aldolase und in allen Fällen die SGR erhöht nachgewiesen wurden.

f) Die Leukosen in einer beginnenden Progredienz zeigten keine signifikanten Enzymerhöhungen wie im unbehandelten Stadium ihrer primären Erkrankung.

g) Bei den übrigen epithelialen Karzinomen findet sich ebenfalls eine deutliche SGR-Ethöhung bei klinisch nachweisbaren Rezidiven und Metastasen. Die Aldolase und die LDH zeigen in einem weitaus geringeren Prozentsatz eine Enzymerhöhung im Serum.

h) Dieser Unterschied tritt bei den übrigen mesenclymalen Tumoren nicht so deutlich in den Vordergrund.

MERTEN und Mitarbeiter (19) haben bei verschiedenen glykolytischen Fermentbestimmungen Unterschiede $z$ wischen Plasma- und Serumwerten gefunden. Aus diesem Grunde haben wir unsere Glutathionreduktase in vergleichenden Untersuchungen im Serum und im Plasma bestimmt. Unsere Erfahrung hat uns aber gezeigt, daß wir mit den Werten der SGR konkretere Aussagen zu machen vermögen.

\section{Literatur}

1. LÜHrs, W., Monats K. ärztl. Fortb. 11, 156 (1960). - 2. Osten, W., Therapeut. Ber. (Bayer) 6, 212 (1962). - 3. LüHRs, W., H. Gummel und J. KindermanN, Zbl. ges. inn. Med. 10, 713 (1955).4. Warburg, O., Weiterentwicklung der zellphysiologischen Methoden, Georg Thieme Verlag, Stuttgart (1962). - 5. Warburg, $O$. und W. Christian, Biochem. Z. 314, 149 (1943). - 6. Hill, B. R. und C. Levi, Cancer Res. 14, 513 (1954). - 7. Mexerhof, O., Pflügers Arch. ges. Physiol. Menschen Tiere 175, 20 (1919). 8. Lührs, W. und E. Negelein, Klin. Wschr. 34, 148 (1956). 9. Sibley, J. und A. L. Lefininger, J. Nat. Cancer Inst., Wash. 9 , 303 (1949); J. biol. Chemistry 177, 859 (1953). - 10. BodANSKY, O. J. biol. Chemistry, 202, 829 (1953). - 11. BRUNs, F. und J. JAKOB,
Klin. Wschr. 32, 1041 (1954). - 12. Bruns, F. und K. Hinsberg, Biochem. Z. 325, 532 (1954). - 13. Rosotto, P. und A. Ruffo, Minerva med., 49, 2145 (1958). - 14. Bodansky, O., Cancer, Philadelphia 10, 865 (1957). - 15. Kaplan, N. O., M. M. Ciotri und F. E. StolzennaAC, J. biol. Chemistry 221, 833 (1956). 16. BrAUN, W., Mitteilungsdienst Ges. Bekämpf. Krebskrankh. Nordrhein-Westfalen 2, 385 (1961). - 17. Mansow, C. und F. WroblewsKr, J. Clin. Invest. 37, 214 (1958). - 18. Kerppola, W. E., A. Nikkilä und E. Pitrkänen, Acta med. Scand. 164, 357 (1959). - 19. SolbaCh, H.-G., A. Engelmardt und R. MerTEN, Mitteilungsdienst Ges. Bekämpf. Krebskrankh. NordrheinWestfalen 2, 375 (1961). 